\title{
SphinX Soft X-ray Spectrophotometer: Science Objectives, Design and Performance ${ }^{1}$
}

\author{
S. Gburek ${ }^{a}$, J. Sylwester ${ }^{a}$, M. Kowalinski ${ }^{a}, J$. Bakala ${ }^{a}$, Z. Kordylewski ${ }^{a}$, P. Podgorski ${ }^{a}$, \\ S. Plocieniak ${ }^{a}$, M. Siarkowski ${ }^{a}$, B. Sylwester ${ }^{a}$, W. Trzebinski ${ }^{a}$, S. V. Kuzin ${ }^{b}$, A. A. Pertsov ${ }^{b}$, \\ Yu. D. Kotov ${ }^{c}$, F. Farnik ${ }^{d}$, F. Reale ${ }^{e}$, and K. J. H. Phillips ${ }^{f}$ \\ a Space Research Center, Polish Academy of Sciences, Poland \\ ${ }^{b}$ P.N. Lebedev Physical Institute of the Russian Academy of Sciences, Russia \\ ${ }^{c}$ National Research Nuclear University "MEPhI", Russia \\ ${ }^{d}$ Astronomical Institute of Academy of Sciences of the Czech Republic, Czech Republic \\ ${ }^{e}$ INAF-G.S. Vaiana Astronomical Observatory, Italy \\ ${ }^{f}$ Mullard Space Science Laboratory, Great Britan
}

Received May 16, 2010

\begin{abstract}
The goals and construction details of a new design Polish-led X-ray spectrophotometer are described. The instrument is aimed to observe emission from entire solar corona and is placed as a separate block within the Russian TESIS X- and EUV complex aboard the CORONAS-PHOTON solar orbiting observatory. SphinX uses silicon PIN diode detectors for high time resolution measurements of the solar spectra in the range $0.8-15 \mathrm{keV}$. Its spectral resolution allows for discerning more than hundred separate energy bands in this range. The instrument dynamic range extends two orders of magnitude below and above these representative for GOES. The relative and absolute accuracy of spectral measurements is expected to be better than few percent, as follows from extensive ground laboratory calibrations.
\end{abstract}

Keywords: solar corona, spectrum, X-ray, abundances, instrumentation, SphinX.

DOI: $10.1134 / \mathrm{S} 0038094611020067$

\section{INTRODUCTION}

Measurements of the X-ray variability of the Sun are of basic importance for studies of the solar activity, physics of flare processes and space weather. The bulk of coronal thermal plasma of temperatures between 1 and $50 \mathrm{MK}$ preferentially contributes to the emission in the soft X-ray band. Observations of the spectral variability in $0.8-15 \mathrm{keV}$ energy range provide the basis for determinations of coronal average properties like temperature, emission measure and related thermodynamic characteristics for non-active corona and flares in particular. For more than 30 years, GOES $X$-ray ion chambers observed the Sun in two spectral bands $0.5-4 \AA$ and $1-8 \AA(3-25 \mathrm{keV}$ and $1.5-12 \mathrm{keV}$ respectively). These nearly continuous measurements performed each three seconds constitute the reference database as concerns contemporary solar activity observations. However they lack the quantitative information at both the low end-and high end-activity ranges. Lack of counts below A class occurs due to not enough detector sensitivity for low X-ray flux. Above X10 class saturation effects affects GOES data and the information about actual flux value is lost.

\footnotetext{
${ }^{1}$ The article was translated by the authors.
}

The other active solar spectrometer presently in orbit (RHESSI, see Lin et al., 2002), have a large area and volume detectors sensitive to emission above $3 \mathrm{keV}$. However, a high level of orbital background prevents quantitative determination of the solar flux at the very low activity conditions, as these present during recent minimum (years 2008-2009).

An illustration of these unfavorable circumstances given the working instruments in orbit is shown in Fig. 1, where we present example measurements taken by GOES and RHESSI for selected time in February 2009, when no flaring activity has been observed for extended time interval.

For purposes of monitoring the solar X-ray emission level during Russian-lead solar space mission CORONAS-PHOTON, it appeared necessary to have a possibility of taking precise measurements of the solar flux level and spectra in the soft X-ray range, also during periods of a very low activity, such as illustrated in Fig. 1.

From this reason, the Wroclaw Solar Physics Division Team at the Polish Space Research Centre (SRC) developed a concept of sensitive spectrophotometer SphinX (Solar photometer in X-rays, Sylwester et al., 2008) which alowed to measure the emission of X-ray 
(a)
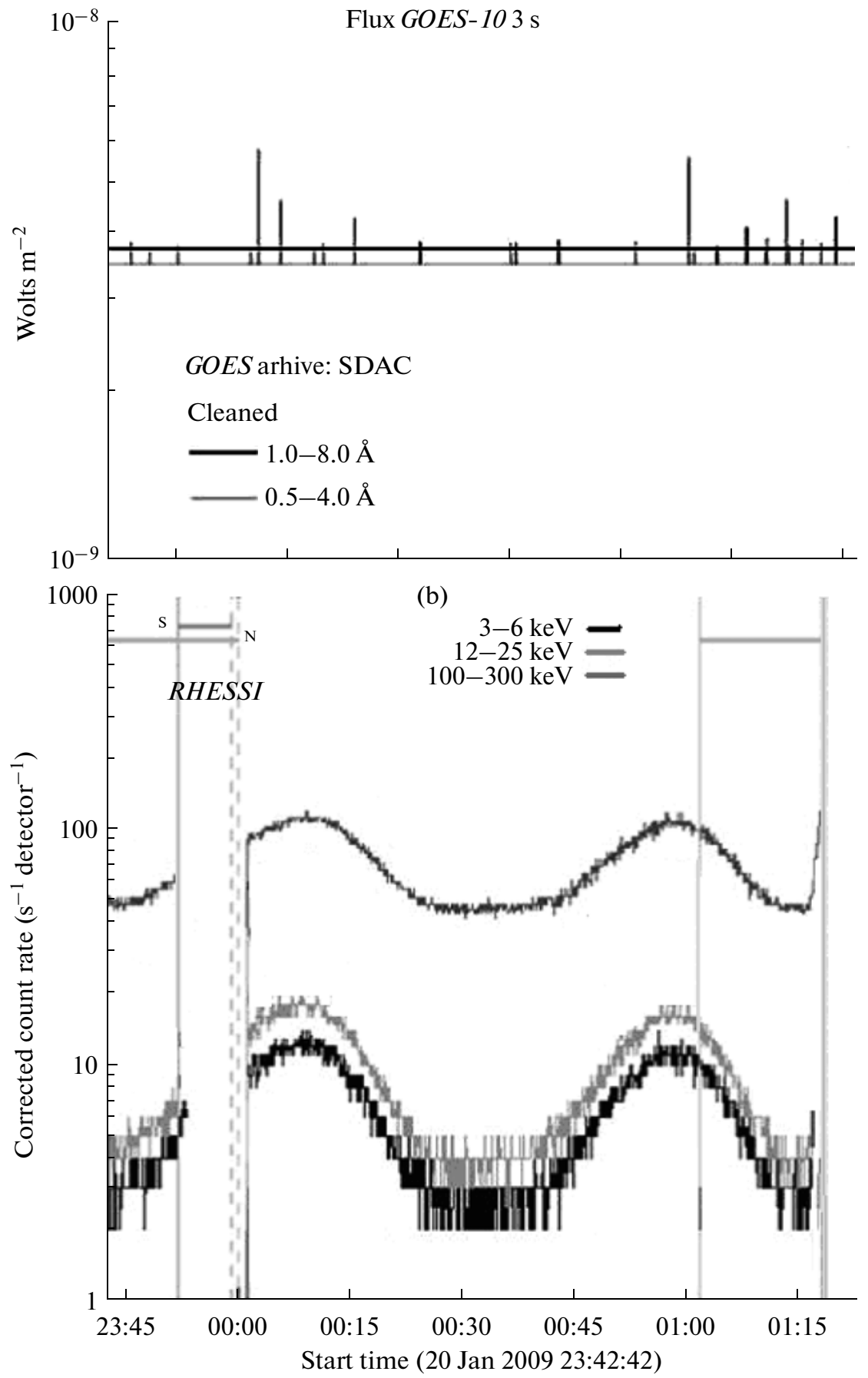

Fig. 1. Example of soft X-ray flux measurements at low solar activity conditions. In the upper panel, the GOES records are drawn. GOES light curve in the Low channel (1-8 ̊̊) keeps constant value at the level of about $3.7 \times 10^{-9} \mathrm{~W} / \mathrm{m}^{2}$ (far below GOES A class) over entire time interval. In GOES High channel $(0.5-4 \AA)$ the light curve also stays mainly flat. Some spikes are only observed in this channel which may come from electronics noises or energetic particles hits into the detector chamber. The rates observed by RHESSI, shown in the bottom panel, are dominated by orbital background.

corona with much increased sensitivity. This spectrometer, using detectors similar as those incorporated into the Indian SOXS experiment (Jain et al., 2005), was accepted as a part of CORONAS-PHOTON sci- entific payload in a very late stage of mission preparation (in 2006, three years before launch) and integrated as a part of the TESIS X- and EUV telescope complex (Kuzin et al., 2006) developed at Lebedev 

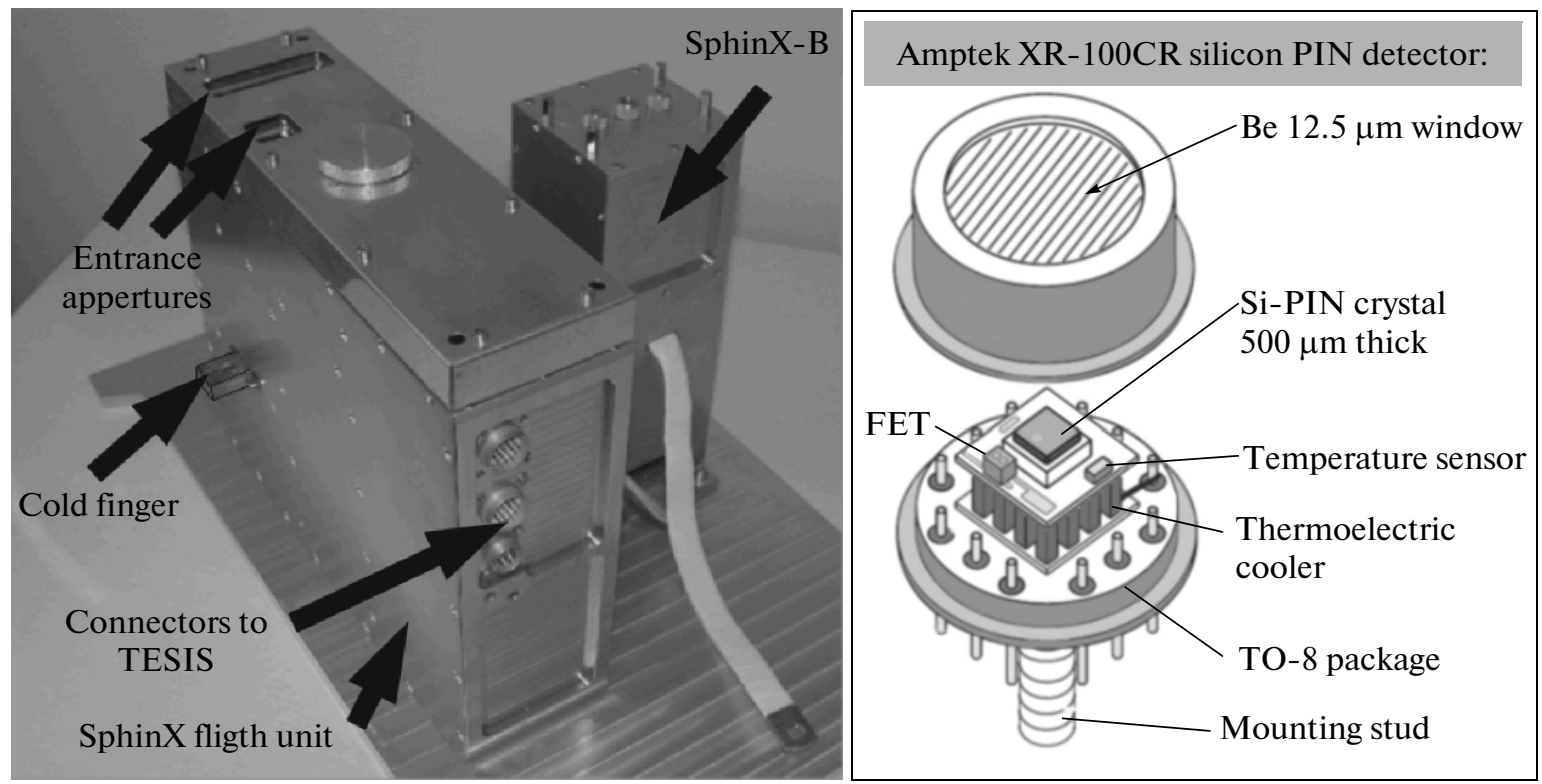

Fig. 2. Left: The photograph of the SphinX flight unit and a smaller SphinX-B box where the flight detectors and electronics have been put for the calibration at the BESSY II Berlin Synchrotron. Due to the constrains of BESSY vacuum test chamber, for purposes of the ground calibrations at the BESSY II, the SphinX spectrophotometric part including detectors D1, D2, and D3 was mounted within a smaller box, also shown in the upper-left part of the photograph. The apertures, connector for cables linking to TESIS as well as heat sink pipe connecting in flight to the external radiator are visible. Right: A scheme of an Amptek detector with individual components indicated. Four such detectors are mounted within SphinX flight unit.

Physical Institute (LPI). Detailed description of CORONAS-PHOTON satellite and its in-flight performance are described (present issue) by Kotov et al. (2010).

The scientific objectives aimed to during the SphinX instrument development phase were the following:

- Investigations of quiet corona heating processes via photon arrival time analysis.

- Studies of soft X-ray oscillations in the period between 1 and $500 \mathrm{~s}$.

- Search for transient ionization plasma effects which may provide a tool for determination of flaring plasma densities.

- Determination of coronal plasma composition and its variability for elements: $\mathrm{Mg}, \mathrm{Al}, \mathrm{Si}, \mathrm{S}, \mathrm{Ar}, \mathrm{Ca}$, and $\mathrm{Fe}$.

- Determination of differential emission measure (DEM) and studies of its time variability.

-Establishment of soft X-ray reference photometric standard with $10 \%$ absolute accuracy.

- Proof of the novel narrow-band photometry (NBF) concept.

- Monitoring of solar X-ray flux in the intensity range covering seven orders of magnitude.

In what follows description of the SphinX instrument is given as well as some results from ground calibrations and data analysis are discussed. Example observations also are shown and discussed.

\section{DESIGN OF THE INSTRUMENT}

SphinX instrument consist of a single blocklarger unit, $27 \times 28 \times 8 \mathrm{~cm}$ illustrated in the photo shown in Fig. 2.

SphinX incorporates four PIN, pure silicon, $500 \mu \mathrm{m}$ thick detectors (Amptek XR-100CR) equipped with Be $12.5 \mu \mathrm{m}$ front filters (see Fig. 2). Beside silicon crystal each detector package contains Peltier cooler, elements of front-end electronics and temperature sensor.

SphinX detectors are mounted on the copper support plate, thermally isolated from the rest of the instrument but joined through the heat sink pipe to external satellite heat radiator. When actively cooled, the detector temperature is approximately $50^{\circ} \mathrm{C}$ below the support plate temperature. SphinX construction details are shown in instrument CAD assembly drawing in Fig. 3.

Three of SphinX detectors named D1, D2, and D3 were placed in main SphinX measurement block designed for solar X-ray spectroscopic and photometric observations. The fourth SphinX detector D4 is used in a special fluorescence measurement unit which is going to be described further on.

Detector D1 used its entire entrance window area $\left(21.50 \mathrm{~mm}^{2}\right)$ during measurements. Over detectors D2 and D3 additional apertures were placed limiting their entrance area to 0.495 and $0.01008 \mathrm{~mm}^{2}$ respectively and thus decreasing their sensitivity to incoming X-ray radiation. 

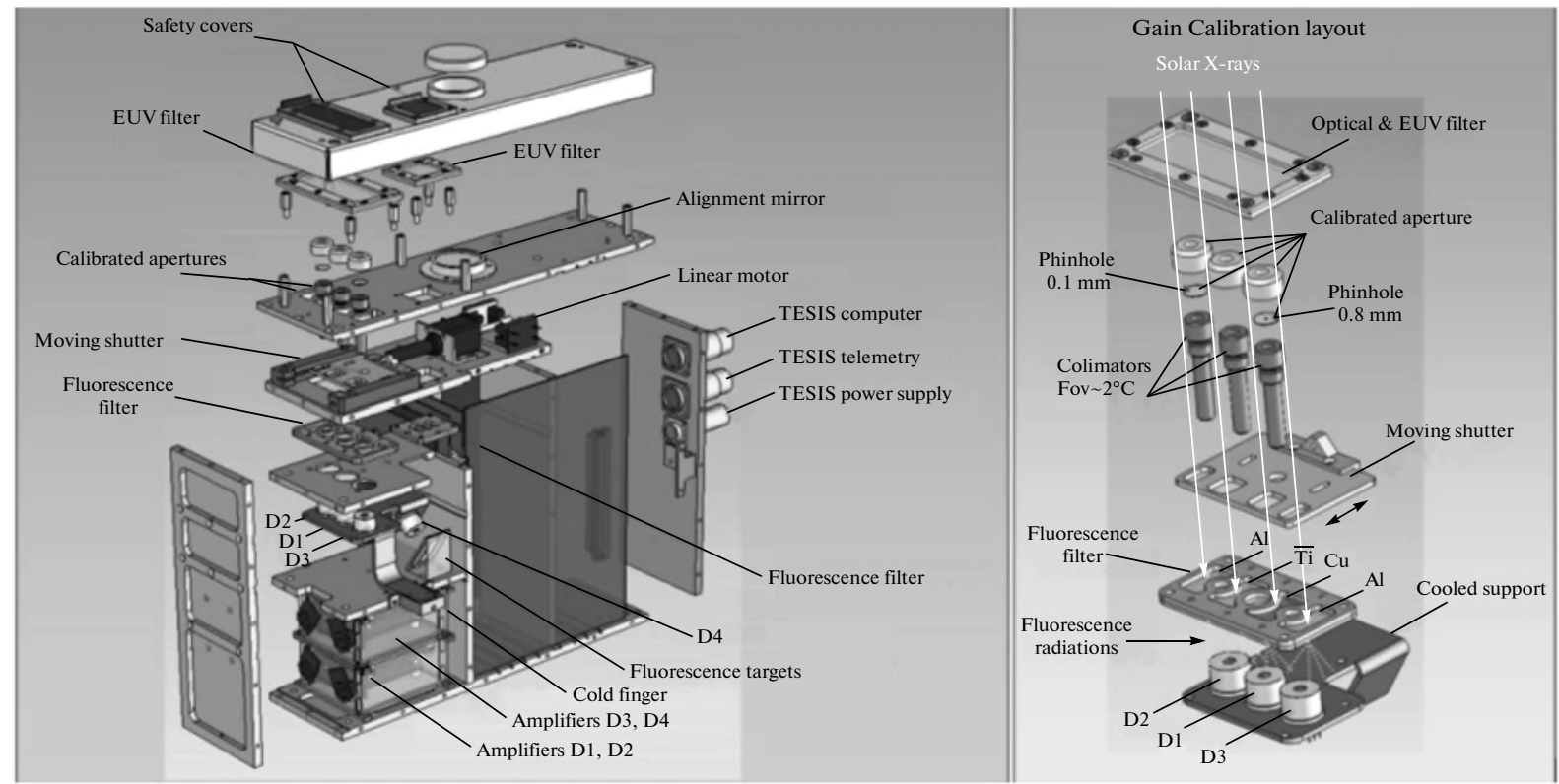

Fig. 3. Left: The assembly drawing of SphinX flight unit. All important construction elements are indicated. Right: Elements of the spectrophotometer main measurement block comprised of three detectors D1, D2, and D3, three collimators and corresponding calibrated apertures. The calibration moving shutter is placed in so-called calibration position, preventing a direct illumination of the detectors. The calibration is performed thanks to detector illumination in characteristic fluorescence lines arising from the back side emission of thin metal foils of three, different atomic weight selected elements ( $\mathrm{Al}, \mathrm{Ti}$, and $\mathrm{Cu}$ ). These foils are partially-transparent to the solar illumination at this shutter position.

Such detector/aperture system is capable of measuring solar X-ray flux for coronal activity levels corresponding to the GOES class as small as 100 times below A1.0 level, through typical quiet conditions and flares up to $G O E S \times 30$ class.

The detector D1 with the highest effective area measures correctly low intensity solar photon fluxes. When the flux gets higher, pileup and saturation start to appear in detector D1 data but measurements with good signal/noise ratio $(S / N)$ are taken by $\mathrm{D} 2$ detector. The aperture over D2 detector is chosen so that it gives measurements of good quality for moderate solar fluxes. The least sensitive D3 detector channel with the smallest aperture can measure with substantial $S / N$ ratio strong solar flux when pileup and saturation effects in detector D2 become significant and detector D1 is completely saturated. Detector D3 should not saturate even at the highest known solar activity level. Thus the dynamic range of the instrument covers seven orders of magnitude. The statistical accuracy of measured rates is better than $1 \%$ for activity conditions above GOES A4.0 level (see Fig. 4).

A moving shutter with a set of openings is placed over detector/aperture assembly (see Fig. 3). The openings are organized the way that it is possible to expose or cover up entry apertures over detectors by moving the shutter. This action is aimed to protect the detectors D1 and D2 against excessive solar flux.

The energy resolution is about $460 \mathrm{eV}$ for $\mathrm{D} 1$ detector channel and $\sim 360 \mathrm{eV}$ for D2 and D3 channels (see table for details), allowing for the solar line groups of $\mathrm{Mg}, \mathrm{Al}, \mathrm{Si}, \mathrm{S}, \mathrm{Ar}, \mathrm{Ca}$, and $\mathrm{Fe}$ to be distinguished above the continuum emission.

SphinX has in-flight calibration capability. The calibration of the detectors is achieved by moving another, so-called calibration shutter into detectors field of view (see the right panel in Fig. 3). By the command from the SphinX computer, the linear motor moves the calibration shutter sideways, closing detectors' direct solar illumination channels and exposing them to indirect fluorescence illumination coming from the back side of thin input transmission filters made of different materials. These three materials are fluorescing at three, precisely known, well separated in energy, monochromatic wavelengths (corresponding to energies for $\mathrm{Al}-1.49 \mathrm{keV}$, Ti-4.51 keV and $\mathrm{Cu}-8.05 \mathrm{keV}$ ) illuminating at the same time all three detectors.

The amplitude positions of the individual peaks are being determined and the on-board computer makes appropriate corrections of amplifiers gains and offsets if large discrepancies between present and initial (preflight calibrated) instrument parameters occur. The widths of the individual peaks contain information on the actual energy resolution of the detectors. Depending on the intensity of the solar illumination, a secondary, pile-up peaks can be resolved, creating thus a possibility to dynamically calibrate the pile-up effect also. After the calibration, the shutter is moved to the home position, where a direct illumination of the detectors through their respective apertures is resumed. In such 
solution of calibration system, the detectors always have three energy-amplitude reference points available and no radioactive sources need to be used. The calibration sequences can be run any time, however it needs the solar activity to be at least at the M1 GOES level, which never happened during the instrument entire mission duration (February-November 2009).

The fourth SphinX detector, measures narrowband fluxes in three wavelength bands: $1.74-2.07,2.50-$ 3.08 , and 7.95-9.54 $\AA$ according to the novel concept presented in Fig. 5. In this narrow-band-filter (NBF) concept, three fluorescing targets illuminate a single PIN detector (D4). The fluorescence radiation is coming from three different pure element targets $(\mathrm{Mg}-1.25 \mathrm{keV}, \mathrm{Ca}-3.69 \mathrm{keV}$, and $\mathrm{Cr}-5.41 \mathrm{keV})$ being illuminated through separate, individual selected input filters made of elements of slightly higher atomic number than their respective targets. Therefore, on the detector, the three emission peaks of different amplitudes are being instantaneously recorded at respective well known energies. Their relative strengths depend on the shape of the solar incident spectrum in three narrow spectral bands and therefore are very sensitive to plasma temperature. We have selected the filter-target materials in order to record the portions of solar spectra containing strong emission lines of iron and magnesium. This would allow us to study transient effects, expected to be seen when emitting plasma conditions vary with time scales less than few seconds. To do so, we need however flare events of the GOES class level above M1. No such event has been seen during SphinX mission unfortunately.

Work on SphinX construction was finished in 2007. Then overall tests of instrument flight unit were performed in order to check if it operates correctly in orbit and can survive severe launch conditions. SphinX was tested in thermal-vacuum chamber in Warsaw, next tests against mechanical/vibration and acoustic damages took place in Prague and finally $\mathrm{X}$-ray calibration tests were performed in Palermo and Berlin.

After completion of all tasks related to development of SphinX, the flight instrument unit was moved to Plesetsk Cosmodrome where it was integrated with the satellite and launched on January 30, 2009. The

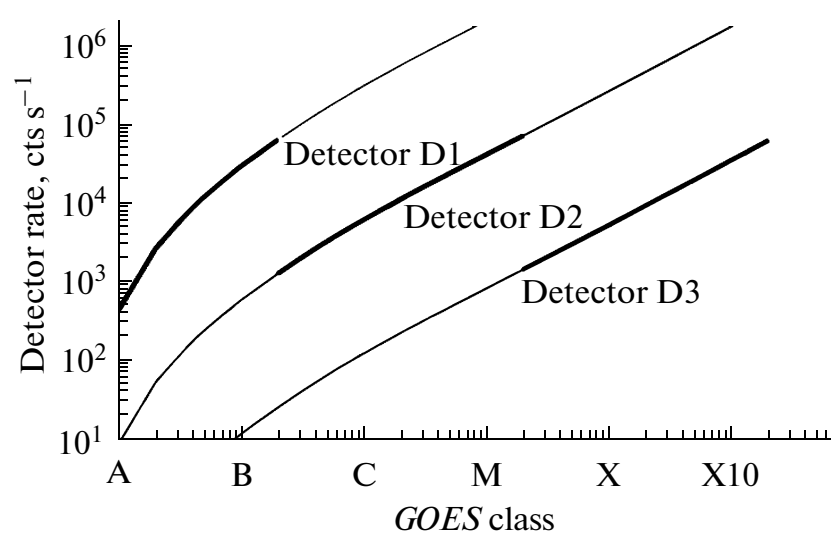

Fig. 4. Expected rates of the photometric signal from the coronal plasma at various levels of solar activity as defined by respective GOES activity classes. The rates were preflight calculated based on typical coronal plasma characteristic: temperatures and emission measures for each GOES class. Detector and electronic saturation effects were not included in the calculations.

satellite and actual location of SphinX within the payload is shown in Fig. 6.

Due to very low general activity of the Sun solar flux during the SphinX mission was too weak to perform the in-flight calibrations and observations in fluorescence channels. Both of these capabilities were discussed above for completeness of description of SphinX construction. On the other hand they are interesting conceptual solutions which significantly extend the instrument observational capabilities and can be implemented in future solar X-ray missions.

\section{CALIBRATION OF THE INSTRUMENT}

The ground calibration of SphinX consisted of three sessions, the first at the XACT facility (Collura et al., 2008) in Palermo (September 2007), and two sessions at the BESSY II Berlin Synchrotron (November 2007 and March 2008).

During XACT test resolution and energy calibration of individual detector channels was tested. It was also possible to make cross-comparison between all SphinX detectors calibration measurements and per-

Basic parameters of SphinX detector measurement channels

\begin{tabular}{l|c|c|c|c}
\hline \multicolumn{1}{c|}{ Detector } & D1 & D2 & D3 & D4 \\
\hline Aperture, $\mathrm{mm}^{2}$ & $21.500^{\mathrm{A}}$ & $0.4947^{\mathrm{S}}$ & $0.01008^{\mathrm{S}}$ & $11.1^{\mathrm{A}}$ \\
Energy FWHM, eV & $480^{\mathrm{B}}$ & $350^{\mathrm{B}}$ & $370^{\mathrm{B}}$ & $290^{\mathrm{P}}$ \\
Shaping time FWHM, $\mu \mathrm{s}$ & $1.25^{\mathrm{e}}$ & $4.17^{\mathrm{e}}$ & $4.17^{\mathrm{e}}$ & $4.17^{\mathrm{e}}$ \\
\hline
\end{tabular}

Notes: A-based on Amptek technical drawings of respective detector, S-measured at SCR Wroclaw under microscope, B-based on BESSY measurements, P-based on Palermo XACT measurements, e-estimated value. 

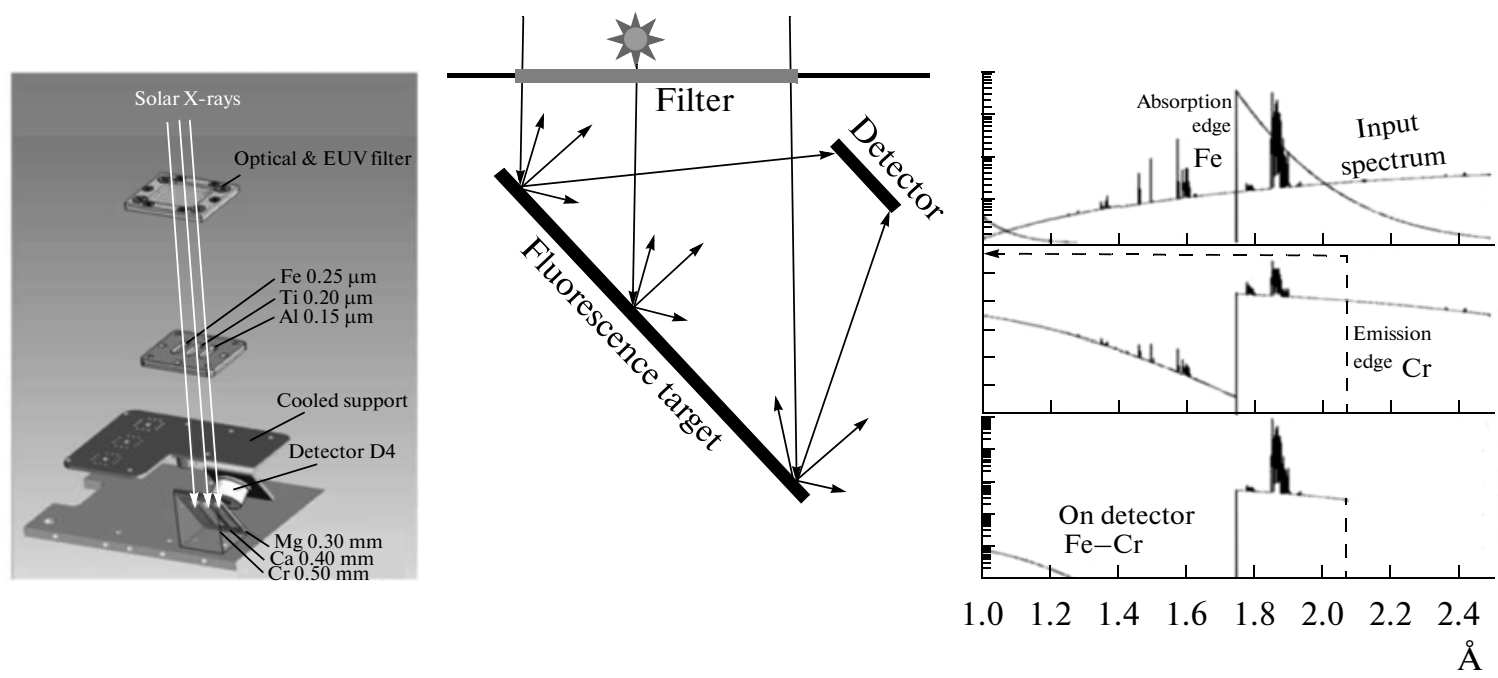

Fig. 5. Left: Actual X-ray optical path of SphinX fluorescence measurement channel including description of all transmission filters and illumination targets. Three pairs of filter-target materials are used in SphinX. Center: The concept of narrow band filter (NBF). Right: solar X-ray spectrum transmitted through the filter contains predominantly the radiation above the wavelength of specific absorption edge. This illuminates the fluorescence target and only those wavelengths below the fluorescence excitation edge contribute to the scattered flux measured by the detector. In respect with, the system acts as the narrow-band filter with precise, naturally defined, sharp energy boundaries.

formance because detectors were illuminated by the same laboratory X-ray source simultaneously.

During the BESSY II tests, accomplished with the help of Physikalisch-Technische Bundesanstalt (PTB) team, the synchrotron beam have been used to calibrate also the response of the three photometric detectors D1, D2, and D3 to illumination from a broad band, rapidly falling with energy, spectra which shapes and intensities were precisely known. An example of such measurement is given in Fig. 7. It was possible to

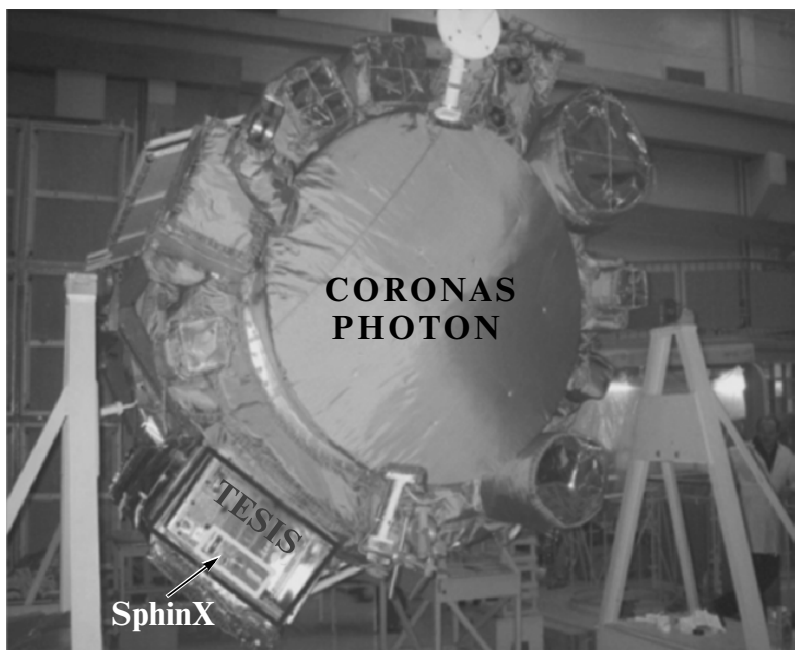

Fig. 6. A photograph of the front of CORONAS-PHOTON mission payload during final launch preparations at the Plesetsk Range. Location of the TESIS instrument complex and the SphinX block are indicated. adjust the synchrotron radiation intensity in a very broad bounds covering a complete dynamic range of SphinX. In addition measurements in monochromatized radiation (with the use of four crystal monochromator) where performed for all SphinX measurement channels in a selected set of energies.

Calibration data obtained at XACT and BESSY II were used to determine SphinX response and efficiency for all detector measurement channels. Details about SphinX calibration will be described in Siarkowski et al. (2010).

\section{DATA ACCESS, EXAMPLE OBSERVATIONS, AND RESULTS}

From the analysis of SphinX calibration data it was found that the optimum number of energy channels for flight SphinX operations is 256. Thus all SphinX detectors used multichannel analyzers with 256 energy bins covering the energy range $0.85-15 \mathrm{keV}$.

During in orbit operations SphinX data were packed by the onboard computer into $8 \mathrm{kB}$ in size memory frames and send to telemetry with cadence of one second usually. Sometimes the cadence was set to five or eight seconds to save on entire telemetry flow volume.

The data received from telemetry were sent via internet to Solar Physics Division of Space Research Center, Polish Academy of Sciences for further processing. Here data were decompressed and divided into binary files containing typically several thousands of telemetry frames and covering a couple of hours of the observation time. These binary files (SphinX level-0 data) are publicly available in IDL native format and 
FITS format. Access to level-0 data is possible via internet catalogues on dedicated SphinX servers. Primary server is located at the Space Research Centre in Wroclaw, Poland ${ }^{2}$, the second in Astronomical Institute of the Academy of Sciences of the Czech Republic in Ondrejov ${ }^{3}$ and the third one in Osservatorio Astronomico di Palermo Giuseppe S. Vaiana in Palermo, Italy ${ }^{4}$. Works on the fourth mirror server in P.N. Lebedev Physical Institute, Russian Academy of Sciences, Moscow are in progress. It is expected that the Moscow server will be operational in the fall of 2010. Each of the level-0 data file can be downloaded directly from Sphinx servers using any web browser.

Analysis and interpretation of level-0 data is possible but necessitates full knowledge of all instrumental effects which are present in data. At the moment all level-0 SphinX data undergo reduction to level-1 data which will be more convenient for scientific analysis purposes. About $40 \%$ of the entire SphinX data repository has been converted to level-1 by June 2010 . Level-1 data will be publicly available on SphinX servers in the second half of 2010.

Alternative, global range access to SphinX data is being organized via Virtual Solar Observatories (VSOs) after reduction off all raw SphinX data to level-1 format. More information on SphinX data formats, reduction and dissemination can be found in Gburek et al. (2010).

During the operation SphinX measured in time stamping mode and spectral mode. In time stamping mode the arrival times and energies of $\mathrm{X}$-ray photons in each detector were registered. Individual photon arrival times were recorded with the $1-2 \mu$ s accuracy.

In the spectral mode photons incident on a detector in a selected number of energy bins were summed and sent to telemetry as histogram of registered events together with the exposure time. Only 256-channel and ancillary 4-channel spectra (so called basic mode observations) were registered during the mission.

Together with SphinX scientific X-ray data, an additional information about start/end time of each measurement and technical block of data were send to telemetry frames, (see also Gburek et al., 2010).

SphinX data coverage is nearly continuous in the time interval from February 20 when SphinX was switched on in orbit till the end of active satellite operation i.e. 29 November, 2009. Only measurements from D1 and D2 detector channels have good $S / N$ ratio in this time period and are useful for analysis. Detectors D3 and D4 registered mostly the noise signal due to low solar X-ray flux during SphinX in orbit operation.

\footnotetext{
${ }^{2}$ http://156.17.94.1/sphinx_catalogue/SphinX_cat_main.html

${ }^{3} \mathrm{http}: / / 147.231 .104 .188 / \mathrm{catalog} /$ SphinX_cat_main.html

${ }^{4}$ http://www.sphinx.astropa.unipa.it/
}

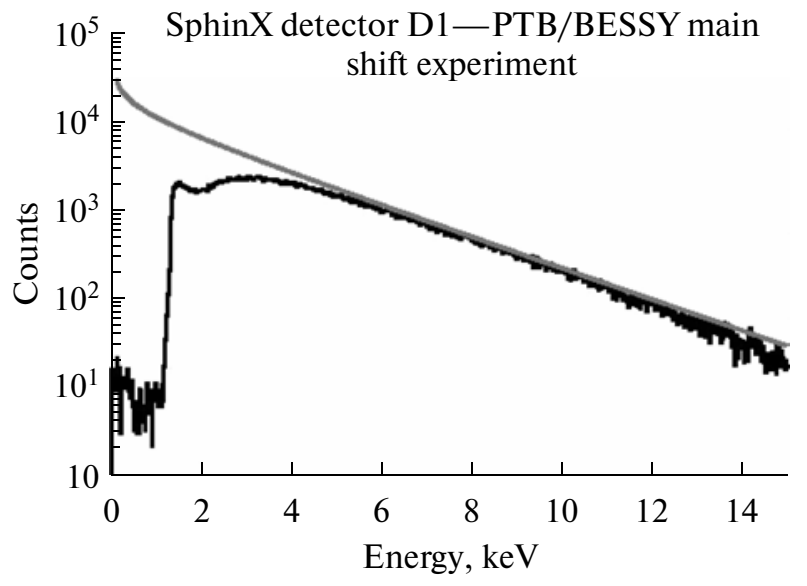

Fig. 7. Direct comparison of the input synchrotron spectrum (gray line) with SphinX measured spectrum in D1 detector channel (given in black line) obtained during BESSY II calibration experiments.

SphinX basic mode observations provide a general information of the observed solar X-ray flux and the information on circumstances in which the instrument operated. In the first channel of basic mode, electronic noises are registered mostly. Rates observed in this channel can be used to check if additional corrections/reductions of data are necessary in a given time interval selected for analysis. In the second and the third channels of basic mode solar broadband $\mathrm{X}$-rays are recorded in energy bands: $1.5-3.0 \mathrm{keV}$ and $3.0-14.9 \mathrm{keV}$ for D1 detector and $1.0-3.0 \mathrm{keV}$ and $3.0-14.9 \mathrm{keV}$ for the rest of the detectors. Lightcurves from both of these channels yield considerable information on solar flux variability and properties and are useful for identification of solar X-ray events (flares and brightennings). These lightcurves may be also used for determination of solar average plasma temperature and emission measure or monitoring intensity of solar flux on longer time intervals.

From the analysis of light curves over long time intervals it is seen that stronger and more variable solar flux shows up when the active regions are present on disk. On the other hand there is observed a presence of some minimum basal level of solar X-ray emission bellow which solar activity never dropped, (see also Gburek et al., 2011).

In the last (the fourth) channel of basic mode observations a strong signal was observed when the satellite was in areas with increased amount of energetic particles. Intensities observed in this channel can be used to identify satellite passages through SAA or polar ovals (see also the next section). Data recorded during such passages usually have an additional spurious contribution from particle hits into detector crystal and need to be rejected from analysis or necessitate special reduction methods. An example of light curves obtained in basic mode observations for detector D1 is shown in Fig. 8. 


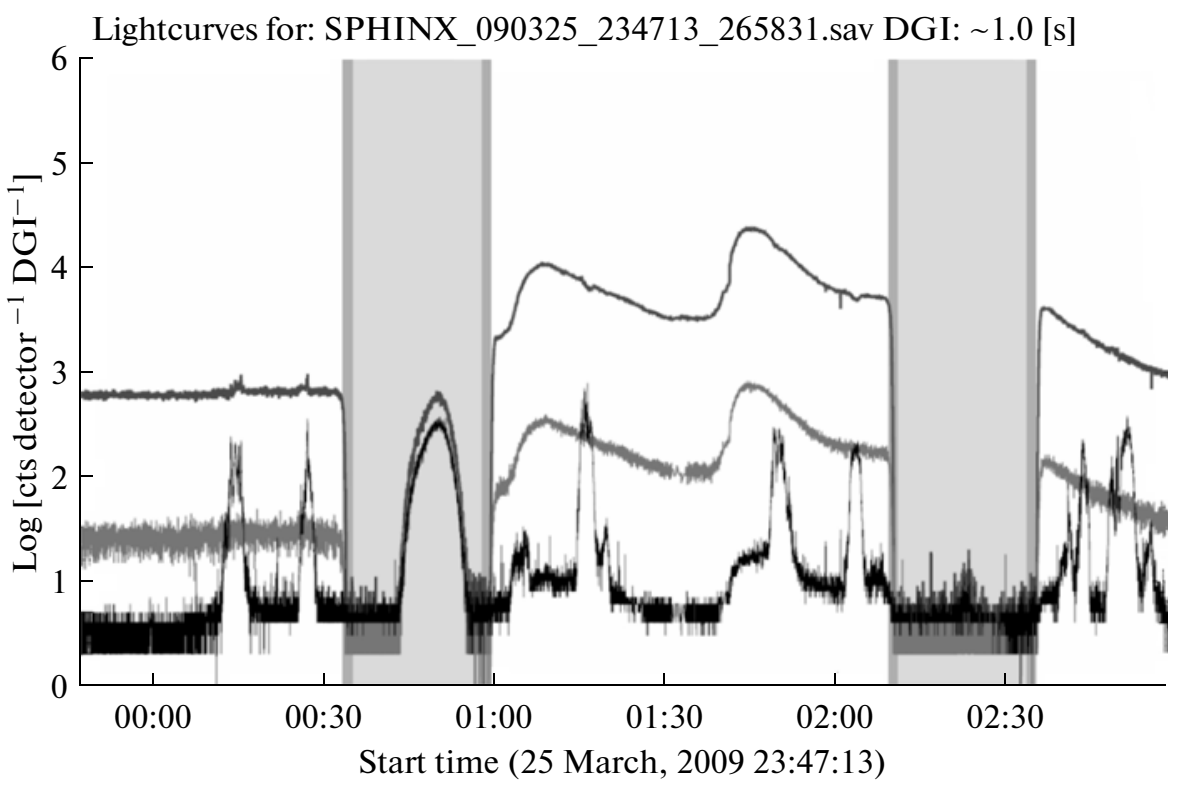

Fig. 8. SphinX lightcurves showing the time variability of solar X-ray flux during the series of flares observed on March $26,2009$. The upper line represents the rate per data gather interval (DGI $=1.0 \mathrm{~s}$ ) as measured using the largest effective area detector D1. Gray vertical strips indicate satellite nights. The rates form D2 detectors are plotted in light-gray line. A particle signal registered in the last channel of basic mode observation in D1 is plotted in black.

SphinX lightcurves from the second and the third channels of basic mode observation allowed to identify about 800 small flare-like events during the active part of the mission. Most of them have maximum intensities below GOES detection threshold. Such small flare-like brightenings have been never observed before with so high spectral and temporal resolution as SphinX offers. For these small events two new classes $S$ (from small, flux range $10^{-9}-10^{-8} \mathrm{~W} / \mathrm{m}^{2}$ ) and $\mathrm{Q}$ (from quiet, flux range $10^{-10}-10^{-9} \mathrm{~W} / \mathrm{m}^{2}$ ) were introduced by Sphinx team to keep consistence with GOES event classification. Full catalog of SphinX observed flare events and brightennings will be made publicly available in the second half of 2010. Other examples of SphinX basic mode observations are given in Gburek et al. (2010).

More advanced SphinX data analysis is possible using spectroscopic observations in which 256 energy channels are available for processing. SphinX spectroscopic observations together with calibration information allow fitting various multi component models in order to determine plasma physical parameters such as the distribution of emission measure with temperature (DEM), verify and compare different solar elements abundance models or ionization equilibrium models used for theoretical calculations of solar spectral shape. Thanks to high sensitivity of SphinX such spectroscopic research is possible even for a very low activity level when no flares, brightening occurred, or even not a single active region was present on the Sun. An example of Sphinx registered spectrum of quiet corona and its analysis is shown in Fig. 9.
The most versatile of SphinX observation modes is time stamping mode in which besides photon energies registered in 256 bins, the information of time arrival of any particular photon also is available. The time stamping mode also allows for additional small corrections of data during reduction procedure. Thus it was a primary mode used in SphinX operation providing most of level-0 data and allowing for their conversion to level-1 format.

Due to instrument operation, the way of sending measurements to telemetry, hardware resets and other reasons the photon stream were not probed continuously in the time stamping mode. Only in short, subDGI time intervals the photons were measured one by one in a sequence.

The number of photons measured in a single continuous sequence vary from a couple of tens of photons up to 1536 photons which is the maximum memory buffer size set for single telemetry frame for a detector. The time gaps between two consecutive measured sequences are typically a fraction of a second long.

All mentioned above (for basic and spectral modes) SphinX scientific analysis directions can be realized using time stamping mode observations. For instance, using this mode, it is possible to investigate solar flux variability on a very short time scales or look for nonMaxwellian plasma processes on the Sun. An example of SphinX observations of quiet corona in time stamping mode is shown in Fig. 10. 


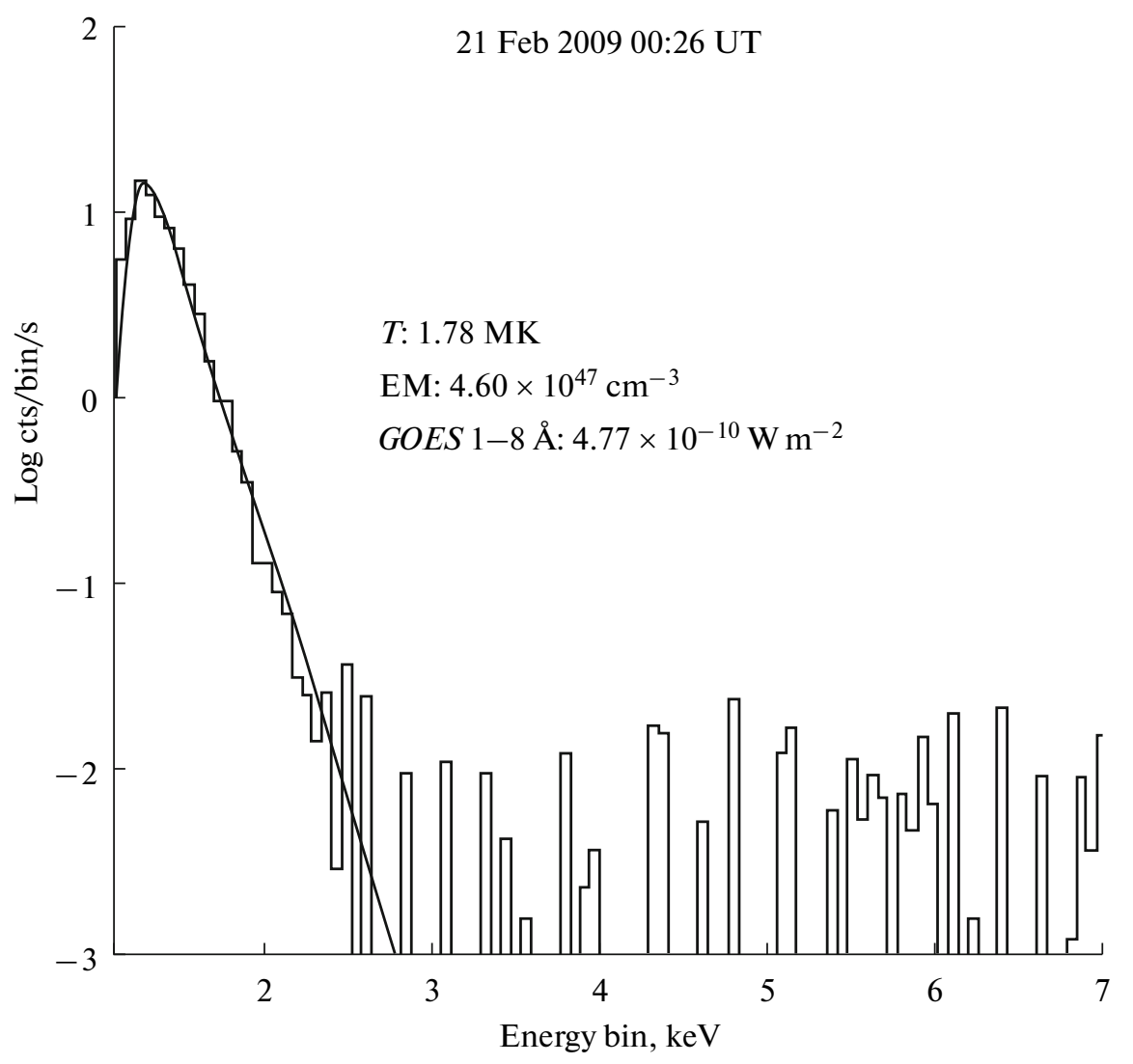

Fig. 9. SphinX X-ray spectra obtained for quiet corona on February 21, 2009. A fit to data obtained in the isothermal approximation is also shown as the smooth continuous line overplotted on measured spectrum. Optimum temperature and emission measure values determined from the fit as well as equivalent flux observed by SphinX in the standard 1-8 Å wavelength band of GOES instrument are indicated in the plot.

\section{THE SATELLITE ORBITAL ENVIRONMENT}

As a consequence of a high inclination of the orbital plane (82.5 degrees), the CORONAS-PHOTON satellite crossed, at the height of $\sim 550 \mathrm{~km}$, regions of increased particle background particularly in South Atlantic Anomaly (SAA) and polar ovals. During these crossings, a higher than average background signal was seen, mostly present in the highest energy bins of all multichannel analyzers connected to individual detectors. This particle signal has been processed for SphinX detector D2 measurement channel and mapped in Fig. 11 to illustrate where the particles may influence the measurements at most.

Outside the areas of increased radiation environment, the particle signal contributes to the spectral signal to very small extent only (less than 0.01 count/s/bin) and can be removed during the process of data reduction.

SphinX is mounted in the Sun-lit portion of the TESIS instrument ${ }^{5}$, as it is illustrated in the photo shown as Fig. 6. It is directly exposed to intense solar illumination. This causes that the temperature inside

\footnotetext{
${ }^{5}$ http://www.tesis.lebedev.ru/
}

the instrument is usually higher than during the ground laboratory calibrations conditions and varies by a few degrees Celsius between day and night portions of the spacecraft orbit. During periods of uninterrupted solar illumination lasting up to three weeks (see Fig. 12), the internal temperature in the instrument raised causing an increase of the thermal noise in the lower energy bins of SphinX measurements. This effect will be corrected during further stages of data reduction.

\section{PLANNED DATA ANALYSIS DIRECTIONS}

Due to unusually weak solar activity in 2009 and, in connection with very low solar flux level over most of SphinX active operation, it was not possible to fulfill all scientific objectives defined during the SphinX instrument development. In particular it was impossible to check the novel narrow-band fluorescence photometry unit. From the same reason in-flight calibrations of the instrument were never done.

The objective of monitoring the solar X-ray flux in the intensity range of seven orders of magnitude has been realized only partly as the solar flux varied over four orders of magnitude during the active operations. 


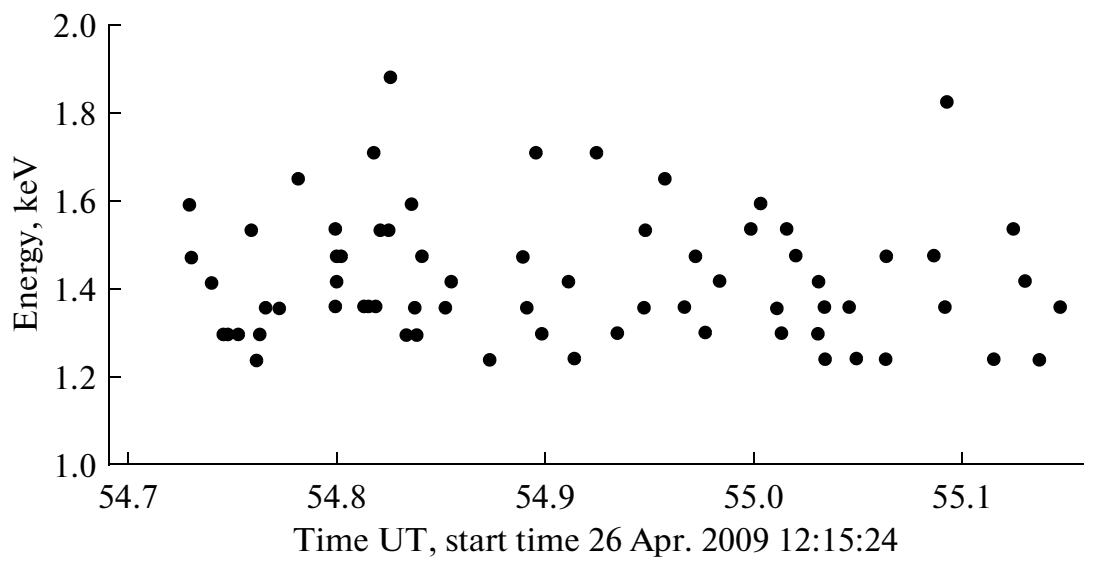

Fig. 10. An X-ray solar photon sequence observed by SphinX in time stamping mode April 26, 2009.

The rest of the objectives, listed in the introduction, can be successfully reached using the collected SphinX data. At present the works on SphinX data focus on:

- Determinations of average solar plasma physical parameters $(T, E M)$ and their time variability.

- Investigations of Solar flares (evolution of characteristics).

- Investigations of early phases of active region emergence.

-Analysis of quiet Sun X-ray flux average properties and characteristic time scales.

-Identification and analysis of very small solar brightennings.

- Search for a presence of a hot plasma components in quiet corona and active regions.
-Analysis of physical plasma parameters and X-ray flux properties in time intervals preceding release of Coronal Mass Ejection.

In addition we will perform studies of dynamics for Earth energetic particle distribution on time scales of weeks to months.

After reduction of all available data to level-1, the SphinX archive will establish the soft X-ray reference photometric standard. Small fluctuations in SphinX measurements occur due to temperature changes mainly. Analysis of SphinX flight data shows that this effect amount to about $10 \%$ of the measured flux value. These fluctuations can be corrected using SphinX technical block data which were send to telemetry along with each X-ray measurement frame. These technical data also allows to trace back the instrument state to its initial calibrated one when all

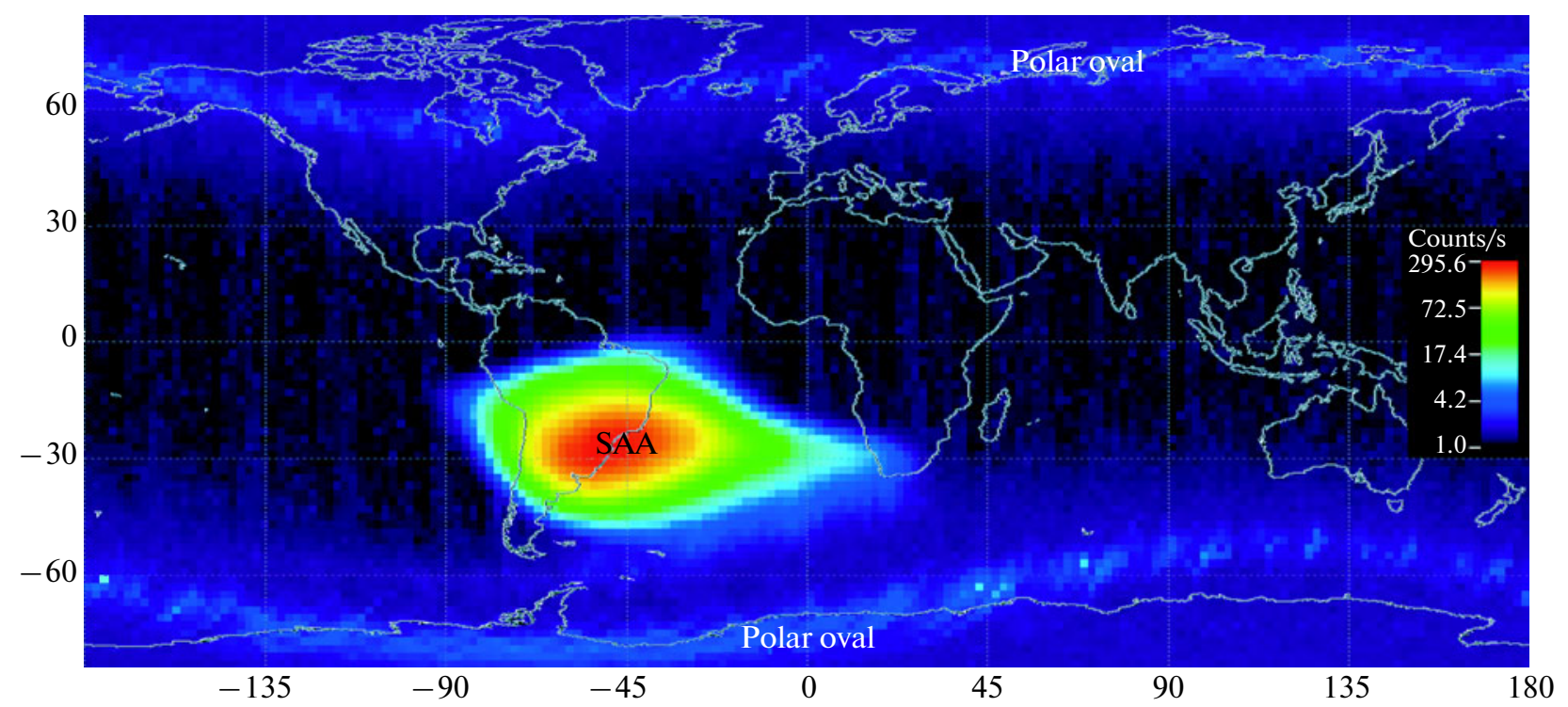

Fig. 11. Map of the reconstructed particle environment as seen in the highest energy bin (bin 255) of SphinX D2 detector measurements. The map represents averaged particle count rates in the $2^{\circ} \times 2^{\circ}$ spatial elements. The averages have been calculated from measurements covering the period between March and October 2009. 


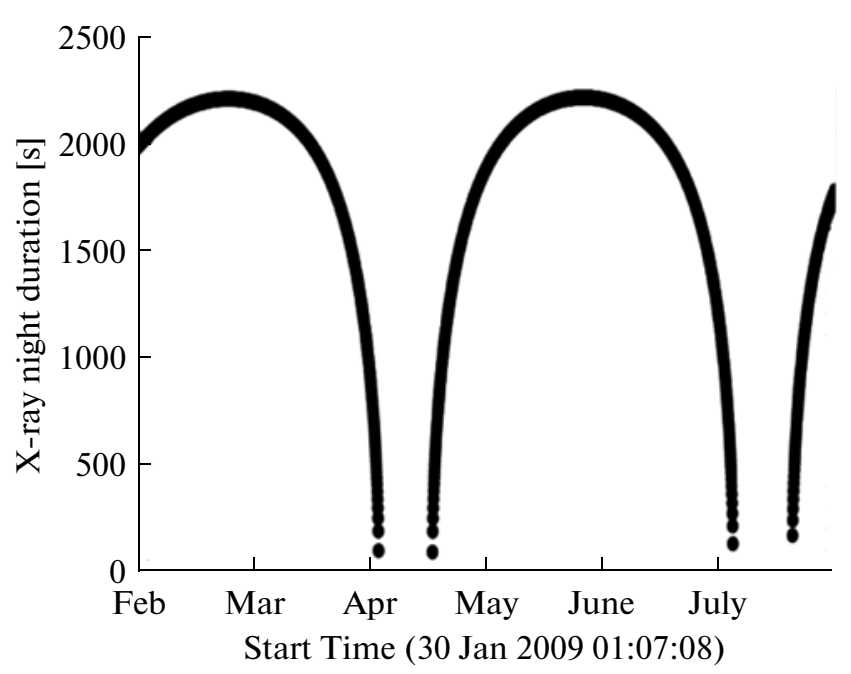

Fig. 12. Duration of spacecraft nights during the first six months of operation.

important instrument characteristics were known. Corrections of all these factors are expected to give the absolute accuracy of flux determinations better than $10 \%$. This will allow us to cross-calibrate measurements between SphinX and the other solar space borne instruments e.g. GOES, RHESSI, SOXS.

\section{CONCLUSION}

SphinX was the most sensitive instrument aimed at measuring the solar soft X-ray radiation and spectra in orbit operating in between February and November 2009. The early interpretation of its results indicates that the Sun is a weak X-ray star during its minimum activity phase, the weakest within seven parsecs stellar neighborhood. The obtained spectra and lightcurves of nonactive corona indicate a presence of basal coronal emission level, nearly unchanged over longer time intervals. Presence of any active region signature causes substantial increase of the X-ray solar flux. Observed, non-active coronal spectra variability points towards a transient nature of this non-active emission over time scale of few minutes. The SphinX instrument enables for the first time to study the physics of microflares and plasma properties of so-called quiet corona.

Work on data reduction and dissemination of level-1 format database continues. The level-1 database will allow for scientific analysis on solar soft X-rays with most of the instrumental effects removed. After completion of level-1 data reduction process the soft X-ray general flux variability will be known with the accuracy of better than $10 \%$. This will enable cross-calibrations of SphinX with the other solar soft X-ray instruments.
Analysis and interpretation of SphinX data is in progress. The activity is focused on determination of basic source plasma properties, identification of flares events and brightenings, search for presence of a hot plasma components in quiet corona and active region plasmas, investigations of active region emergence and decay processes, and statistical analyses important for Space Weather applications.

\section{ACKNOWLEDGMENT}

The Polish Ministry of Education and Science grant no. N203 381736 is acknowledged for financial support of the SphinX project which is being developed within the Polish Academy of Sciences and Russian Academy of Sciences bi-lateral agreement on cooperation in space research. This work was partially supported by the Russian Foundation for Basic Research (project no. 08-02-01301-a), program for fundamental research Physical Department of the Russian Academy of Sciences "Processes in Solar System Plasma". The research leading to these results has received funding from the European Commission's Seventh Framework Programme (FP7/2007-2013) under the grant agreement no. 218816 (SOTERIA project, www.SOTERIA-space.eu).

\section{REFERENCES}

Collura, A., Barbera, M., Varisco, S., et al., Calibration of the SphinX Experiment at the XACT Facility in Palermo, Proc. SPIE-Int. Soc. Opt. Eng., 2008, vol. 7011, p. $70112 \mathrm{U}$.

Gburek, S., Siarkowski, M., Kempa, A., et al., Soft Solar X-ray Radiation Dynamics during Current Minimum of Solar Activity according to SphinX Observations, Astron. Vestn., 2011, vol. 45, no. 2, p. 186.

Jain, R., Dave, H., Shah, A.B., et al., Solar X-ray Spectrometer (SOXS) Mission on Board GSAT2 Indian Spacecraft: The Low-Energy Payload, Sol. Phys., 2005, vol. 227, p. 89.

Kotov, Yu.D., et al., Astron. Vestn., 2011, vol. 45, no. 2.

Kuzin, S.V., Zhitnik, I.A., Bogachev, S.A., et al., Solar Activity and Its Magnetic Origin, Proc. 233rd Symp. Int. Astron. Union, Cairo, Egypt, 2006, Bothmer, V. and Abdel Hady, A., Eds., Cambridge: Univ. Press, 2006, pp. 449-451.

Lin, R.P., Dennis, B.R., Hurford, G.J., et al., The Reuven Ramaty High-Energy Solar Spectroscopic Imager (RHESSI), Sol. Phys., 2002, vol. 210, p. 3.

Siarkowski, M., Gburek, S., Kowalinski, M., et al., Interpretation of the Ground Calibration of SphinX Detectors Using BESSY Synchrotron Beams, (in preparation).

Sylwester, J., Kuzin, S., Kotov, Yu.D., et al., SphinX: A Fast Solar Photometer in X-rays, J. Astrophys. Astron., 2008, vol. 29, pp. 339-343. 